\title{
SPIO Nanoparticle
}

National Cancer Institute

\section{Source}

National Cancer Institute. SPIO Nanoparticle. NCI Thesaurus. Code C68566.

Nanoparticles that have been functionalized with chemical amine groups and dextran.

These particles have been utilized as magnetic resonance imaging agents and as probes

to investivate thrombosis and tumor vasculature in conjunction with a CREKA peptide

(cys-arg-glu-lys-ala). 